Aus der Chirurgischen Abteilung des Städtischen Krankenhauses I in Hannover. (Oberarzt: Prof. Dr. H. Schlange.)

\section{Ueber isolierte, subcutane Fissuren der langen Röhrenknochen.}

\section{Von Dr. Karl Försterling, Assistenzarzt.}

Im Städtischen Krankenhause I in Hannover sind in letzter Zeit mehrere Male Knochenverletzungen beobachtet worden, die bisher in den chirurgischen Lehrbüchern und den hierher gehörigen Veröffentlichungen nur kurz erwähnt wurden, ohne dab weiter auf ihre eventuelle Bedeutung eingegangen wurde. Es handelte sich um isolierte, subcutane Fissuren in der Diaphyse von Röhrenknochen. Ich möchte zuerst zwei Fälle, die recht typisch sind, beschreiben.

Fall 1. J. W., ein siebenjähriger, mittelkräftiger Knabe, dem eine Bettmatratze auf den rechten Unterschenkel gefallen war. H hatte sofort ïber Schmerzen geklagt und nicht mehr gehen können

Es fand sich an der Vorderfläche der Tibia, an Fig. 1. der Grenze ihres mittleren und unteren Drittels

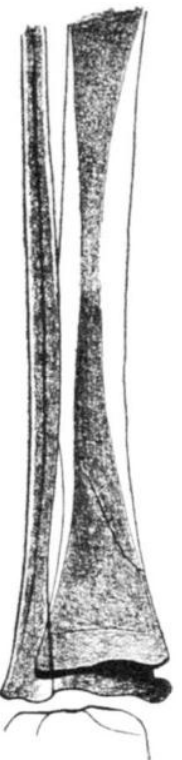
dere leichte umschriebene sehr druckempfindliche Schwellung; eine Veränderung am Knochen, insbesondere an der vorderen Tibiakante, war nicht zu fühlen. Bei Untersuclıung auf abnorme Beweglichkeit war rielleicht ein geringes Federn dej Knochens vorhanden; jedoch war dieses nicht deutlich. Dagegen klagte das Kind dabei sehr iiber heftige Schmerzen. Ferner war der Knabe nicht $z_{11}$ bewegen mit dem Finbe aufzutreten stellte man ihn auf die Firde, so beugte er das rechte Bein im Knie. Die Röntgenaufnahme vo vorn zeigte nun eine feine, $3 \mathrm{~cm}$ oberhalb de Fußgelenkes an der Innenseite der Tibia beginnende Fissur des Knochens, die sich reichlich $3 \mathrm{~cm}$ weit schräg nach oben außen erstreckte. dabei gut die Hälite des Knochens durchsetzend, und nach kurzem, senkrechtem Verlanf mitten im Knochen endigte. Patient w urde nach vier Wochen geheilt entlassen; auf der Vorderfläche der Tibia war eine flache, eben fiillbare Soliwellung zu tasten. Im Röntgenbild war der Befund der gleiche; kein Callus zu sehen (cf. Fig. 1)

Fall 2. G. S., zwei Jahre alter, gut entwickelter Knabe. Patient ist vor fünf Tagen einige Treppenstufen hinabgefallen. Die Eltern bringen das Kind, weil es immer noch nicht wieder laufen will und über Schmerzen im linken Bein klagt. Es findet sich im unteren Teile des mittleren Drittels der Tibia auf deren Vorderfläche eine zirkumskripte. geringfügige, teigige Schwellung, wie man sie nach einfachen Kontusionen an der vorderen Unterschenkelfläche täglich sehen kann. Eine Veränderung am Knochen war nicht vorhanden, vor allem war nicht die geringste abnorme Beweglichkeit nachzuweisen. Dagegen schien die Untersuchung sehr schmerzhaft zı sein. Auch dieses Kind war nicht dazu zu bringen, auf das verletzte Bein zu treten. Obwohl es sehr nach der in nur kurzer Entfernung stehenden Mutter verlangte und die Arme nach ibr ausstreckte, so hielt es doch stets das linke Bein im Knie angezogen und berührte mit dem Fuße nie den Frdboden.

Die Röntgenaufnahme von vorn ergab dak es sich um eine Fissur der Tibia handelte die in deren Mitte an der Innenseite begann und schräg nach außen-unten $3 \mathrm{~cm}$ weit ver lief bis zur Corticalis der Außenseite, ohne diese jedoch zu durchsetzen. Bei seitlicher Aufnahme war von der ganzen Verletzung nichts zu sehen. Nach etwa 14 Tagen trat der Knabe wieder auf und blieb schmerzfrei (cf. Fig. 2).

Bevor ich weiter auf das Thema eingehe, möchte ich feststellen, was unter einer Fissur zu verstehen ist. Mit Bruns ${ }^{1}$ fasse ich als solche einen unvollständigen Längs-, Schräg- oder Torsionsbruch auf, bei dem weder eine Dislokation der Bruchenden

1) Bruns, Die Lehre von den Knochenbrūchen. Deutsche Chlrurgie, Lieferung $27, \mathrm{~S} .84$. noch eine Deformierung des Knochens noch ein irgendwie erhebliches Klaffen des Bruchspaltes vorhanden ist. Die Infraktion zeigt im Gegensatz dazu ein hrlaffen des Bruchspaltes und eine Dislocatio ad axin oder eine Kompression des Knochens.

Daß Fissuren am Knochen vorkommen, ist nun durchaus nichts neues, sondern sogar schon sehr lange bekannt. Nach Bruns (l. c. S. 91) stammt die erste Mitteilung über diesen sogenannten „Kleckbruch“ aus dem Jahre 1620 von Wülttz, der ihn für sehr gefährlich hielt. Fast ausnahmslos handelte es sich dabei jedoch um Fissuren, die mit Frakturen kompliziert waren und von diesen aus sich in den Knochen erstrecktell. Anders dagegen verhält es sich mit den isolierten und unkomplizierten Diaphysenfissuren; sie sind Raritäten, da sie ja bisher nur durch Obduktion, sei es am Lebenden, sei es am Toten. festgestellt werden konnten. Bruns, der die Knochenfissuren (l. c. S. 81-91) zieınlich ausführlich behandelt. kann im ganzen nur drei Fälle aus der Literatur zusammenstellen und fügt zwei aus der Tübinger Klinik hinzu; im ganzen also fünf. eine wahrlich recht geringe Zahl bei der großen Menge andrer Knochenverletzungen.

König ${ }^{1}$ schreibt, daß die Fissuren durch die Röntgenstrahlen ilıre Bestätigung gefunden lätten und fiihrt da\%ı tinen von Reinlı $\operatorname{ardt}^{3}$ ) beschriebenen Fall an. Diese Verletzung dürfte aber eher als Fraktur aufzufassen scin, da sie ja den Knochen in seiner ganzen Dicke durchsetzt. [Eine Anzall solcher Querfrakturen ohne Dislokation, die vielleicht olne ZerreiBung des Periosts verlaufen sind, hat letzthin $H$ enning ${ }^{3}$ ) veriffentliclt.] Tillmanns reht auf isolierte Fissuren iiberhaupt nicht eill, solndern erwähnt nur die mit Frakturen verbundenen. $H$ of $f^{5}{ }^{5}$ fihhrt bei den Tibiafissuren nur zwei, auch bei Bruns beschriebene Fälle an und bezeichnet sie als Seltenlieiten.

Am Lebenden ist die sichere Diagnose einer einfachen. subcutanen, isolierten Diaphysenfissur (und nur un solche soll es sich hier handeln) vor Entdeckung der Röntgenstrahlen wohl überhaupt nicht zu stellen möglich gewesen. Alle diese Fälle sind eben als Kontusionen betrachtet und behandelt worden. Hierdurch wurden die Patienten meist auch wohl nicht geschädigt, da derartige, immerhin doch leicht zu nennende Knochenverletzungen schnell und ohne dauernden Nachteil heilen. Anders ist es schon, wenn sich eine solche Fissur bis in ein benachbartes Gelenk fortsetzt. In solchem Falle könnte sie wohl durch Hervorrufen von Wucherungen am Gelenkknorpel eventuell die Veranlassung zu Bewegungsstörungen abgeben

Aber für die Praxis sind auch die einfachen, isolierten Diaphysenfissuren denn doch nicht ganz ohne Bedeutung, wie es vielleicht von vornherein scheinen könnte. Dadurch daß ihr Vorkommen als galnicht so selten sichergestellt ist, wird vielleicht manche erhebliche Funktionsstörung und lange Heilungsdauer nach einer Kontusion erklärlich. Llenn solange nicht eine knöcherne Vereinigung des Knochensprungs eingetreten ist, bleibt natürlich jede Belastung desselben, sei es durch Druck oder Zug, schmerzhaft. So findet auch wohl mancher Unfallverletzte, der anfangs auf Grund des geringen objektiven Befundes als ein arger Uebertreiber angesehen wurde, dann noch seine Rechtfertigung, wenn man eine solche kleine Fissur im Knochen annimmt.

Wann soll nun der Arzt überhaupt an einen solchen Knochensprung denken? Die Heftigkeit des vorausgegangenen Trauma spielt dabei nur eine geringe Rolle. Wichtig dagegen ist, daß stets eine Funktionsstörung stärkerer .trt an dem betroffenen Gliede vorhanden ist, die zu dem objektiven Befunde im krassen Widerspruch steht. Die Extremität ist neist völlig gebrauchsunfähig wie bei einer Fraktur. Das übrige Bild ist jedoch nur das einer einfachen Kontusion: geringe, umschriebene Schwellung und Schmerzhaftigkeit, Freisein der nächstliegenden Gelenke, nichts von Krepitation, Dislokation oder abnormer Beweglichkeit. Selbstverständlich ist damit die Diagnose noch nicht gesichert; nur hat man dann an eine Fissur zu denken und daraufhin eine Röntgenuntersuchung

l) König, Lehrbuch der spez. Chirurgie 1900 , Bd. 3, S. 682. - 2) Reinhardt Deutsche Zeitschrift für Chirurgie Bd, 50, S. 328. - 3) Henning, Deutsche Zeitschrif für Chirurgie Bd. 75, S. 262. - 4) Tillmanns, Lehrbuch der allgeneinen Chirurgle 1899, S. 522. - 5) Hoffa, Lehrbuch der Frakturen und Luxationen 1896, S. 621 
vorzunehmen. Jedoch darf man sich nicht mit einer einfachen Durchleuchtung begnügen, da die Spalten so klein sind, daß sie auf dem Schirm nicht zutagetreten. Nein, es müssen Aufnahmen gemacht werden, und zwar von zwei Seiten, wie das bei der Röntgenuntersuchung der Frakturen schon häufig betont worden ist. Unter Umständen ist nämlich die Fissur bei Durchstrahlung nur von einer Seite nicht zu sehen, wie der oben beschriebene zweite Fall zeigt. Sobald die verletzte Corticalispartie bei einem nicht runden Knochen, wie es z. B. die Tibia ist, nur direkt seitlich von den Strahlen getroffen wird, ist der Spalt nicht zu sehen, da ja jede Dislokation fehlt und die vom Röntgenlicht zu durchdringende Knochenschicht überall die gleiche ist oder wenigstens durch den Sprung nicht verändert wird.

Einen solchen Fall beschreibt auch Goldammer:') Bei Aufnabme des Unterschenkels von vorn war cine Knochenverletzung nicht nachweisbar, dagegen eine Fissur der Tibia bei seitlicher Durchstrahlung. Nach Heinrich" ${ }^{2}$ sollen ja sogar Knochenverletzungen mit nachifolgender stärkeser Callusbildung vorkommen, dic im Röntgenbild ïberhaujt nicht nachweisbar sind. Diese Beobachtung wird wohl mit Verbesserung ier Technik in der Röntgenographie sich kaum noch bäufiger wiederholen.

Auf die Theorie der Entstehung solcher Fissuren will ich nicht näher eingehen. Es handelt sich, wie schon oben angedeutet, um die Vorstufe der Längs-, Schräg- und Spiralbrüche. Die Kontinuitätstrennung ist in einem Teile der Corticalis erfolgt; infolge der Elastizität des unversehrten Knochens wird jedoch das Klaffen des Spaltes und Dislokation verhindert. Durch Biegen kann man eine Diastase der Spaltränder hervorrufen und bei Steigerung der angewandten Kraft eine vollkommene Infraktion, resp. Fraktur zustande bringen. Es mahnt dies, bei der Untersuchung nicht zu rücksichtslos vorzugehen, damit nicht einmal eine Fissur in eine Fraktur verwandelt wird.

Bezüglich der Häufigkeit der Fissuren glaube ich, daß sie nicht so selten sind, als man bisher nachweisen konnte. Bei genauer Kontrolle der mit Funktionsstörungen schwerer Art einhergehenden Kontusionen durch Röntgenaufnahmen wird man sie häufiger finden. Denn mit dem Eintreten der eventuellen Callusbildung ind nur durch diese konnte man doch früher am Lebenden die Fissur nachträglich feststellen) verscliwinden auch die Beschwerden, und der Patient entzieht sich der ärztlichen Beobachtung. Aulierdern konnte aber die Knochenverdickung ja auch auf einer Periostitis beruhen, die durch die kiontusion hervorgerufell war.

Prognostisch sind die isolierten Diaphysenfissuren wohl absolut günstig. Sie heilen schnell und ohne dauernde Funktionsstörung unter Ruhigstellung des verletzten Gliedes.

Meinem verehrten Chet, Herrn Prof. H. Schlange, danke ich auch an dieser Stelle bestens fiir die Ueberlassung des Materials und die Anregung zur Arbeit.

1) Golda mm er. Deutsche militärärztliche Zeitschrift 1903, S. 568. - 2) Heinrich. Beitrag zur Frage der Diagnose von Knochenverletzungen durch dss Róntgenbild. [nauguraldissertation, Greifswald 1903. 